\title{
On the Non-Common Neighbourhood Energy of Graphs
}

\author{
Ahmad N. Al-Kenani1, Anwar Alwardi'2, Omar A. Al-Attas' \\ ${ }^{1}$ Department of Mathematics, King Abdulaziz University, Jeddah, Saudi Arabia \\ ${ }^{2}$ Department of Studies in Mathematics, University of Mysore, Mysore, India \\ Email: aalkenani10@hotmail.com, a wardi@hotmail.com, Omar alattas30@hotmail.com
}

Received 20 May 2015; accepted 30 June 2015; published 3 July 2015

Copyright (C) 2015 by authors and Scientific Research Publishing Inc.

This work is licensed under the Creative Commons Attribution International License (CC BY).

http://creativecommons.org/licenses/by/4.0/

cC) (i) Open Access

\begin{abstract}
In this paper, we introduce a new type of graph energy called the non-common-neighborhood energy $E_{\mathrm{NCN}}(G)$, NCN-energy for some standard graphs is obtained and an upper bound for $E_{\mathrm{NCN}}(G)$ is found when $G$ is a strongly regular graph. Also the relation between common neighbourhood energy and non-common neighbourhood energy of a graph is established.
\end{abstract}

Keywords

NCN-Eigenvalues (of Graph), NCN-Energy (of Graph), NCN-Adjacency Matrix (of Graph)

\section{Introduction}

Let $G$ be a simple graph with $n$ vertices, and let $\boldsymbol{A}=\left\|a_{i j}\right\|$ be its adjacency matrix. The eigenvalues $\lambda_{1}, \lambda_{2}, \cdots, \lambda_{n}$ of $\boldsymbol{A}$ are the (ordinary) eigenvalues of the graph $G[1]$. Since $\boldsymbol{A}$ is a symmetric matrix with zero trace, these eigenvalues are real with sum equal to zero.

The energy of the graph $G$ is defined [2] as the sum of the absolute values of its eigenvalues:

$$
E(G)=\sum_{i=1}^{n}\left|\lambda_{i}\right|
$$

Details on the theory of graph energy can be found in the reviews [3]-[5], whereas details on its chemical applications in the book [6] and in the review [7]. Let $G$ be simple graph with vertex set $V(G)=\left\{v_{1}, v_{2}, \cdots, v_{n}\right\}$. For $i \neq j$, the common neighborhood of the the vertices $v_{i}$ and $v_{j}$, denoted by $\Gamma\left(v_{i}, v_{j}\right)$, is the set of vertices, different from $v_{i}$ and $v_{j}$, which are adjacent to both $v_{i}$ and $v_{j}$. The common-neighborhood matrix of $G$ is then $\mathbf{C N}=\mathbf{C N}(G)=\left\|\gamma_{i j}\right\|$, where 


$$
\gamma_{i j}= \begin{cases}\left|\Gamma\left\{v_{i}, v_{j}\right\}\right| & \text { if } i \neq j \\ 0 & \text { otherwise. }\end{cases}
$$

The common-neighborhood energy (or, shorter, CN-energy) of the graph $G$ is

$$
E_{\mathrm{CN}}=E_{\mathrm{CN}}(G)=\sum_{i=1}^{n}\left|\gamma_{i}\right| \text {. }
$$

where $\gamma_{1}, \gamma_{2}, \cdots, \gamma_{n}$ are the eigenvalues of the $\mathbf{C N}(G)$, for more details about CN-energy, see [9]

Theorem 1. [8] For almost all $n$-vertex graphs

$$
E(G)=\left(\frac{4}{3 \pi}+o(1)\right) n^{3 / 2}
$$

Theorem 1 immediately implies that almost all graphs are hyperenergetic, making any further search for them pointless.

In what follows we shall need a few auxiliary results.

Lemma 1. [1] Let $G$ be a connected k-regular graph with $n$ vertices and $k \geq 3$. Let $k, \lambda_{2}, \cdots, \lambda_{n}$ be its eigenvalues. Then the eigenvalues of the line graph of $G$ are $2 k-2, \lambda_{2}+k-2, \cdots, \lambda_{n}+k-2$, and -2 with multiplicity $n(k-2) / 2$.

Lemma 2. [1] Let $G$ be a graph with an adjacency matrix $A$ and with eigenvalues $\lambda_{1}, \lambda_{2}, \cdots, \lambda_{p}$, then the $\operatorname{det} A=\prod_{i=1}^{p} \lambda_{i}$, for any polynomial $P(x), P(\lambda)$ is an eigenvalue of $P(A)$ and hence $\operatorname{det} P(A)=\prod_{i=1}^{p} P\left(\lambda_{i}\right)$.

Corollary 1. [9] Let $G$ be a connected $k$-regular graph and let $k, \lambda_{2}, \cdots, \lambda_{n}$ be its eigenvalues.

1) The common-neighborhood eigenvalues of the complement of $G$ are

$$
(n-k-1)(n-k-2), \lambda_{2}^{2}+2 \lambda_{2}-n+k+2, \cdots, \lambda_{n}^{2}+2 \lambda_{n}-n+k+2
$$

2) The common-neighborhood eigenvalues of the line graph $L(G)$ of $G$ are

$$
4 k^{2}-10 k+6, \lambda_{2}^{2}+(2 k-4) \lambda_{2}+k^{2}-6 k+6, \cdots, \lambda_{n}^{2}+(2 k-4) \lambda_{n}+k^{2}-6 k+6,6-2 k
$$

where the CN-eigenvalue $6-2 k$ has multiplicity $n(k-2) / 2$.

Definition. A strongly regular graph with parameters $(n, k, \lambda, \mu)$ is a $k$-regular graph with $n$ vertices, such that any two adjacent vertices have $\lambda$ common neighbors, and any two non-adjacent vertices have $\mu$ common neighbors.

\section{Non-Common Neighbourhood Energy of Graphs}

Definition. Let $G$ be simple graph with vertex set $V(G)=\left\{v_{1}, v_{2}, \cdots, v_{n}\right\}$. For $i \neq j$, the non-common neighborhood set of the the vertices $v_{i}$ and $v_{j}$, denoted by $\Gamma^{\prime}\left(v_{i}, v_{j}\right)$, is the set of vertices, different from $v_{i}$ and $v_{j}$, which are not adjacent to both $v_{i}$ and $v_{j}$. The non-common neighborhood matrix of $G$ is then

$\mathbf{N C N}=\mathbf{N C N}(G)=\left\|a_{i j}\right\|$, where

$$
a_{i j}=\left\{\begin{array}{lc}
\left|\Gamma^{\prime}\left\{v_{i}, v_{j}\right\}\right| & i \neq j \\
0 & \text { otherwise. }
\end{array}\right.
$$

According to the above definition, the non-common neighborhood matrix is a real symmetric $n \times n$ matrix. Therefore its eigenvalues $\gamma_{1}, \gamma_{2}, \cdots, \gamma_{n}$ are real numbers. Since the trace of $\mathbf{N C N}(G)$ is zero, the sum of its eigenvalues is also equal to zero. the eigenvalues $\gamma_{1}, \gamma_{2}, \cdots, \gamma_{n}$ of the matrix NCN $(G)$ are called the CNCeigenvalues of $G$

Definition. The non-common neighborhood energy (or, shorter, CNC-energy) of the graph $G$ is

$$
E_{\mathrm{NCN}}=E_{\mathrm{NCN}}(G)=\sum_{i=1}^{n}\left|\gamma_{i}\right| \text {. }
$$

We will Denote by $\boldsymbol{I}_{n}$ the unit matrix of order $n$, and by $\boldsymbol{J}_{n}$ the square matrix of order $n$ whose all elements are equal to unity. Let further $\mathbf{0}$ stand for a matrix (or pertinent dimension) whose all elements are 
equal to zero.

Proposition 2. $E_{\mathrm{NCN}}\left(K_{n}\right)=E\left(\overline{K_{n}}\right)=0$, where $K_{n}$ is the complete graph of order $n$.

Proof. Observing that $\mathrm{NCN}\left(K_{n}\right)=A\left(\overline{K_{n}}\right)$, we get $E_{\mathrm{NCN}}\left(K_{n}\right)=E\left(\overline{K_{n}}\right)=0$.

Proposition 3. $E_{N C N}\left(K_{a, b}\right)=2[(a-1)(a-2)+(b-1)(b-2)]$, where $K_{a, b}$ is the complete bipartite graph of order $a+b$.

Proof. First observe that if the vertices of $K_{a, b}$ are labeled so that all vertices $v_{1}, \cdots, v_{a}$ are adjacent to all vertices $v_{a+1}, \cdots, v_{a+b}$, then

$$
\operatorname{NCN}\left(K_{a, b}\right)=\left(\begin{array}{cc}
\mathbf{0} & (a-2)\left(\boldsymbol{J}_{a}-\boldsymbol{I}_{a}\right) \\
(b-2)\left(\boldsymbol{J}_{b}-\boldsymbol{I}_{b}\right) & \mathbf{0}
\end{array}\right) .
$$

Observing that $\boldsymbol{J}_{a}-\boldsymbol{I}_{a}=\boldsymbol{A}\left(K_{a}\right)$ and $\boldsymbol{J}_{b}-\boldsymbol{I}_{b}=\boldsymbol{A}\left(K_{b}\right)$, we have

$$
\operatorname{NCN}\left(K_{a, b}\right)=\left(\begin{array}{cc}
\mathbf{0} & (a-2) \boldsymbol{A}\left(K_{a}\right) \\
(b-2) \boldsymbol{A}\left(K_{b}\right) & \mathbf{0}
\end{array}\right)
$$

Which implies $E_{\mathrm{NCN}}\left(K_{a, b}\right)=(a-2) E\left(K_{a}\right)+(b-2) E\left(K_{b}\right)=2[(a-1)(a-2)+(b-1)(b-2)]$.

Corollary 2. $E_{\mathrm{NCN}}\left(P_{3}\right)=E_{\mathrm{NCN}}\left(K_{2,2}\right)=E_{\mathrm{NCN}}\left(P_{2}\right)=0$

Proposition 4. $E_{\mathrm{NCN}}\left(K_{a, a}\right)=(n-2)(n-4)$, where $K_{a, a}$ is the complete bipartite graph of order $a+a=n$.

Proposition 5. For any totally disconnected graph $\overline{K_{n}}, E_{\mathrm{NCN}} \overline{K_{n}}=2(n-1)(n-2)$.

Proof. Observing that for any two vertices $u$ and $v$ in $\mathbf{N C N}\left(\overline{K_{n}}\right)$ there are $n-2$ vertices not adjacent to both vertices $u$ and $v$. Therefore $\mathbf{N C N}\left(\overline{K_{n}}\right)=(n-2) \boldsymbol{A}\left(K_{n}\right)$, where $\boldsymbol{A}\left(K_{n}\right)$ is the adjacency matrix of the complete graph with $n$ vertices. Hence, $E_{\mathrm{NCN}}\left(\overline{\left(K_{n}\right)}\right)=(n-2) E\left(K_{n}\right)=2(n-1)(n-2)$.

The complete multipartite graph $K_{p_{1}, p_{2}, \cdots, p_{r}}$ is a graph on $n=\sum_{i=1}^{r} p_{i}$ vertices. The set of vertices is partitioned into parts of cardinalities $p_{1}, p_{2}, \cdots, p_{r}$; an edge joins two vertices if and only if they belong to different parts. Thus $K_{1,1, \cdots, 1}$ is the complete graph $K_{n}$. In the following proposition we get the CNC-energy of the multipartite graph $K_{p_{1}, p_{2}, \cdots, p_{r}}$. Then,

Proposition 6. Let $K_{p_{1}, p_{2}, \cdots, p_{r}}$ be The complete multipartite graph on $n=\sum_{i=1}^{r} p_{i}$ vertices, where $p_{i} \geq 3$.

$$
E_{\mathrm{NCN}}\left(K_{p_{1}, p_{2}, \cdots, p_{r}}\right)=\sum_{i=1}^{r}\left(\left(p_{i}-2\right) E\left(K_{p_{i}}\right)\right)=\sum_{i=1}^{r}\left(E_{\mathrm{CN}}\left(K_{p_{i}}\right)\right) .
$$

Proof. Let $G=K_{p_{1}, p_{2}, \cdots, p_{r}}$ be a complete multipartite graph with $n=\sum_{i=1}^{r} p_{i}$ vertices. From the definition of complete multipartite graph we observe for any two distinct vertices $v_{s}, v_{t}$ if they belong to the same partite set $S_{p_{i}}$ with $\left|S_{p_{i}}\right|=p_{i}$, then $\Gamma^{\prime}\left(v_{s}, v_{t}\right)=p_{i}-2$. But if the two vertices belongs to different partite sets we have $\Gamma^{\prime}\left(v_{s}, v_{t}\right)=0$. Hence the NCN-matrix of $K_{p_{1}, p_{2}, \cdots, p_{r}}$ is of the following form.

$$
\left(\begin{array}{ccccc}
\left(p_{1}-2\right) \boldsymbol{A}\left(K_{p_{1}}\right) & 0 & 0 & \cdots & 0 \\
0 & \left(p_{2}-2\right) \boldsymbol{A}\left(K_{p_{2}}\right) & 0 & \cdots & 0 \\
0 & 0 & \left(p_{3}-2\right) \boldsymbol{A}\left(K_{p_{3}}\right) & \cdots & 0 \\
\vdots & \vdots & \vdots & \ddots & \vdots \\
0 & 0 & 0 & \cdots & \left(p_{r}-2\right) \boldsymbol{A}\left(K_{p_{r}}\right)
\end{array}\right) \text {, }
$$

where $\boldsymbol{A}\left(K_{p_{i}}\right) ; i=1,2, \cdots, r$ is the adjacency matrix of the complete graphs $K_{p_{i}} ; i=1,2, \cdots, r$. Hence,

$$
\begin{aligned}
E_{\mathrm{NCN}}\left(K_{p_{1}, p_{2}, \cdots, p_{r}}\right) & =\left(p_{1}-2\right) E\left(K_{p_{1}}\right)+\left(p_{2}-2\right) E\left(K_{p_{2}}\right)+\cdots+\left(p_{r}-2\right) E\left(K_{p_{r}}\right) \\
& =\sum_{i=1}^{r}\left(\left(p_{i}-2\right) E\left(K_{p_{i}}\right)\right)=\sum_{i=1}^{r}\left(E_{\mathrm{CN}}\left(K_{p_{i}}\right)\right) .
\end{aligned}
$$


Corollary 3. For graph $G=K_{r \times m}$ we have,

$$
E_{\mathrm{NCN}}\left(K_{r \times m}\right)=2 r(m-1)(m-2) .
$$

Corollary 4. For any cocktail party graph $G$ which is the complement of $(n / 2) K_{2}$,

$$
E_{\mathrm{NCN}}(G)=0 .
$$

The proof of the following result is straightforward.

Proposition 7. If the graph $G$ consists of (disconnected) components $G_{1}, G_{2}, \cdots, G_{p}$, then

$$
E_{\mathrm{NCN}}(G)=E_{\mathrm{NCN}}\left(G_{1}\right)+E_{\mathrm{NCN}}\left(G_{2}\right)+\cdots+E_{\mathrm{NCN}}\left(G_{p}\right) .
$$

Theorem 8. Let $G$ be a graph on $n$ vertices, and let $A(G)$ is the adjacency matrix of $G$, and $B(G)=\left\|b_{i, j}\right\|$, where

$$
b_{i, j}=\left\{\begin{array}{lc}
\operatorname{deg}\left(v_{i}\right)+\operatorname{deg}\left(v_{j}\right), & \text { if } i \neq j \text { and } v_{i} v_{j} \in E ; \\
\operatorname{deg}\left(v_{i}\right)+\operatorname{deg}\left(v_{j}\right)+2, & \text { if } i \neq j \text { and } v_{i} v_{j} \notin E ; \\
0, & \text { otherwise. }
\end{array}\right.
$$

and Let $\boldsymbol{D}(G)=\operatorname{diag}\left[\operatorname{deg}\left(v_{1}\right), \operatorname{deg}\left(v_{2}\right), \cdots, \operatorname{deg}\left(v_{n}\right)\right]$. Then,

$$
\mathbf{N C N}(G)=n\left(\boldsymbol{J}_{n}-\boldsymbol{I}_{n}\right)-\boldsymbol{B}(G)+\boldsymbol{A}(G)^{2}-\boldsymbol{D}(G) .
$$

Proof. Since $(\operatorname{NCN}(G))_{i j}$ is equal to size of the set $\Gamma^{\prime}\left(v_{i}, v_{j}\right)$. Therefore $\mathbf{N C N}(G)=n\left(\boldsymbol{J}_{n}-\boldsymbol{I}_{n}\right)-\boldsymbol{B}(G)+\mathbf{C N}(G)$ and as we know that $\mathbf{C N}(G)=\boldsymbol{A}(G)^{2}-\boldsymbol{D}(G)$. Hence

$$
\mathbf{N C N}(G)=n\left(\boldsymbol{J}_{n}-\boldsymbol{I}_{n}\right)-\boldsymbol{B}(G)+\boldsymbol{A}(G)^{2}-\mathbf{D}(G) .
$$

Lemma 3. Let $G=(V, E)$ be k-regular graph and $\boldsymbol{B}(G)=\left\|b_{i, j}\right\|$, where

$$
b_{i, j}=\left\{\begin{array}{lc}
\operatorname{deg}\left(v_{i}\right)+\operatorname{deg}\left(v_{j}\right), & \text { if } i \neq j \text { and } v_{i} v_{j} \in E ; \\
\operatorname{deg}\left(v_{i}\right)+\operatorname{deg}\left(v_{j}\right)+2, & \text { if } i \neq j \text { and } v_{i} v_{j} \notin E ; \\
0, & \text { otherwise. }
\end{array}\right.
$$

Then

$$
\boldsymbol{B}(G)=(2 k+2)\left(\boldsymbol{J}_{n}-\boldsymbol{I}_{n}\right)-2 \boldsymbol{A}(G) \text {. }
$$

Proof. Observing that if $G=(V, E)$ is $k$-regular, then $\boldsymbol{B}(G)=\left\|b_{i, j}\right\|$, where

$$
b_{i, j}=\left\{\begin{array}{lc}
2 k, & \text { if } i \neq j \text { and } v_{i} v_{j} \in E ; \\
2 k+2, & \text { if } i \neq j \text { and } v_{i} v_{j} \notin E ; \\
0, & \text { otherwise. }
\end{array}\right.
$$

Therefore,

$$
\boldsymbol{B}=2 k \boldsymbol{A}(G)+(2 k+2)\left(\boldsymbol{J}_{n}-\boldsymbol{I}_{n}-\boldsymbol{A}(G)\right) .
$$

Hence

$$
\boldsymbol{B}=(2 k+2)\left(\boldsymbol{J}_{n}-\boldsymbol{I}_{n}\right)-2 \boldsymbol{A}(G) .
$$

Proposition 9. For any k-regular graph $G$,

$$
\mathbf{N C N}(G)=\boldsymbol{A}(G)^{2}+2 \boldsymbol{A}(G)+(n-2 k-2) \boldsymbol{J}_{n}-(n-k-2) \boldsymbol{I}_{n} .
$$

Proof. By Theorem 8 and Lemma 3, we have

$$
\mathbf{N C N}(G)=\boldsymbol{A}^{2}+2 \boldsymbol{A}(G)+\left(\boldsymbol{J}_{n}-\boldsymbol{I}_{n}\right)-(2 k+2)\left(\boldsymbol{J}_{n}-\boldsymbol{I}_{n}\right)-k \boldsymbol{I}_{n} .
$$


Hence

$$
\mathbf{N C N}(G)=\boldsymbol{A}(G)^{2}+2 \boldsymbol{A}(G)+(n-2 k-2) \boldsymbol{J}_{n}-(n-k-2) \boldsymbol{I}_{n} .
$$

Theorem 10. For any graph $G, E_{\mathrm{NCN}}(G)=E_{\mathrm{CN}}(\bar{G})$.

Proof. Since $\operatorname{NCN}(G)_{i j}$ for $i \neq j$ is the number of vertices which not adjacent to both $v_{i}$ and $v_{j}$ and it is equal to the number of vertices which adjacent to both $v_{i}$ and $v_{j}$ in $\bar{G}$, that means $(\mathbf{N C N}(G))_{i j}=(\mathbf{C N}(\bar{G}))_{i j}$.

Theorem 11. Let $G$ be a connected $k$-regular graph with eigenvalues $k, \lambda_{2}, \cdots, \lambda_{n}$. Then the NCN-eigenvalues of $G$ are $(k-n+1)(k-n+2),\left(\lambda_{2}\right)^{2}+2 \lambda_{2}-n+k+2, \cdots,\left(\lambda_{n}\right)^{2}+2 \lambda_{n}-n+k+2$.

Proof. Theorem 11 follows from Proposition 8 and Lemma 2 or by applying Theorem 10 and Corollary 1

Theorem 12. Let $G$ be a connected k-regular graph and let $k, \lambda_{2}, \cdots, \lambda_{n}$ be its eigenvalues. Then The NCNeigenvalues of the line graph $L(G)$ of $G$ are

$$
2(k-n+1)(k-n+2)-2,\left(\lambda_{2}\right)^{2}+2 \lambda_{2}-n+2 k, \cdots,\left(\lambda_{n}\right)^{2}+2 \lambda_{2}-n+2 k .
$$

Proof. Theorem 12 follows from Proposition 8 and Lemma 2 or by applying Theorem 10 and Corollary 1

Theorem 13. For any connected graph $G, E_{\mathrm{NCN}}(G)=0$ if and only if $G$ is complete multi bipartite graph $K_{n_{1}, n_{2}, \cdots, n_{m}}$ for some positive integer $m \geq 2$, where $n_{i} \leq 2$ for $i=1,2, \cdots, m$.

Proof. Let $G \cong K_{n_{1}, n_{2}, \cdots, n_{m}}$ for some positive integer $m \geq 2$, where $n_{i} \leq 2$ for $i=1,2, \cdots, m$. Suppose that $u$ and $v$ any two vertices in $G$, if $u$ and $v$ adjacent, then does not exists any vertex in $G$ which is not adjacent to both of $u$ and $v$, similarly if $u$ and $v$ are not adjacent that means $\operatorname{NCN}(G)$ is zero matrix. Therefore $E_{\mathrm{NCN}}(G)=0$ and Corollary 2 are spacial cases of multi bipartite graph $K_{n_{1}, n_{2}, \cdots, n_{m}}$ for some positive integer $m \geq 2$, where $n_{i} \leq 2$ for $i=1,2, \cdots, m$.

Conversely, if $G$ is connected graph and $E_{\mathrm{NCN}}(G)=0$, then by Theorem $10 E_{\mathrm{CN}}(\bar{G})=0$. Therefore $G \cong \overline{\left(\alpha K_{1} \cup \beta K_{2}\right)}$ for some positive integers $\alpha, \beta \geq 0$. Hence $G$ is complete multi bipartite graph $K_{n_{1}, n_{2}, \cdots, n_{m}}$ for some positive integer $m \geq 2$, where $n_{i} \leq 2$ for $i=1,2, \cdots, m$.

Lemma 4. If $G$ is a strongly regular graph with parameters $(n, k, \lambda, \mu)$, then

$$
\sum_{i=1}^{n}\left|\gamma_{i}\right|^{2}=n\left[k(n-2 k+\lambda)^{2}-(k-n+1)(n-2 k+\mu-2)^{2}\right]
$$

Proof. If $v_{i}$ and $v_{j}$ are adjacent vertices of $G$, then $\gamma_{i j}=n-2 k+\lambda$. If $v_{i}$ and $v_{j}$ are non-adjacent vertices of $G$, then $\gamma_{i j}=p-2 k+\mu-2$. Since $G$ has $n k / 2$ pairs of adjacent vertices, and $\left(\begin{array}{l}n \\ 2\end{array}\right)-n k / 2$ pairs of non-adjacent vertices,

$$
\begin{aligned}
\sum_{i=1}^{n}\left|\gamma_{i}\right|^{2} & =\operatorname{Tr}(\mathbf{N C N}(G))^{2}=\sum_{i=1}^{n} \sum_{j=1}^{n} \gamma_{i j} \gamma_{j i}=\sum_{i=1}^{n} \sum_{j=1}^{n}\left(\gamma_{i j}\right)^{2} \\
& =2\left[\frac{n k}{2}\right](n-2 k+\lambda)^{2}+2\left[\left(\begin{array}{l}
n \\
2
\end{array}\right)-\frac{n k}{2}\right](n-2 k+\mu-2)^{2},
\end{aligned}
$$

from which Equation (1) follows straightforwardly.

Theorem 14. If $G$ is a strongly regular graph with parameters $(n, k, \lambda, \mu)$, then

$$
\begin{aligned}
& E_{\mathrm{NCN}}(G) \leq(k-n+1)(k-n+2) \\
& +\sqrt{(n-1)\left[n\left[k(n-2 k+\lambda)^{2}-(k-n+1)(n-2 k+\mu-2)^{2}\right]-(n-1)[(k-n+1)(k-n+2)]^{2}\right]} .
\end{aligned}
$$

Proof. follows an idea first used by Koolen and Moulton [10] [11]. Let $\gamma_{1}, \gamma_{2}, \cdots, \gamma_{n}$ be the common neighborhood eigenvalues of $G$, and let $\gamma_{1}$ be the greatest eigenvalue. Because the greatest ordinary eigenvalue of $G$ is equal to $k$, by Theorem $14, \gamma_{1}=(k-n+1)(k-n+2)$.

The Cauchy-Schwarz inequality states that if $\left(a_{1}, a_{2}, \cdots, a_{n}\right)$ and $\left(b_{1}, b_{2}, \cdots, b_{n}\right)$ are $n$-vectors, then 


$$
\left(\sum_{i=1}^{n} a_{i} b_{i}\right)^{2} \leq\left(\sum_{i=1}^{n} a_{i}^{2}\right)\left(\sum_{i=1}^{n} b_{i}^{2}\right)
$$

Now, by setting $a_{i}=1$ and $b_{i}=\left|\gamma_{i}\right|, i=2,3, \cdots, n$, in the above inequality, we obtain

$$
\left(\sum_{i=2}^{n}\left|\gamma_{i}\right|\right)^{2} \leq\left(\sum_{i=2}^{n} 1^{2}\right)\left(\sum_{i=2}^{n}\left|\gamma_{i}\right|^{2}\right)
$$

Therefore

$$
\sum_{i=2}^{n}\left|\gamma_{i}\right| \leq \sqrt{(n-1) \sum_{i=2}^{n}\left|\gamma_{i}\right|^{2}}
$$

i.e.,

$$
\sum_{i=1}^{n}\left|\gamma_{i}\right|-(k-n+1)(k-n+2) \leq \sqrt{(n-1)\left[\sum_{i=1}^{n}\left|\gamma_{i}\right|^{2}-[(k-n+1)(k-n+2)]^{2}\right]}
$$

i.e.,

$$
E_{\mathrm{NCN}}(G) \leq(k-n+1)(k-n+2)+\sqrt{(n-1)\left[\sum_{i=1}^{n}\left|\gamma_{i}\right|^{2}-[(k-n+1)(k-n+2)]^{2}\right]} .
$$

By using Lemma 4,

$$
\begin{aligned}
& E_{\mathrm{NCN}}(G) \leq(k-n+1)(k-n+2) \\
& +\sqrt{(n-1)\left[n\left[k(n-2 k+\lambda)^{2}-(k-n+1)(n-2 k+\mu-2)^{2}\right]-(n-1)[(k-n+1)(k-n+2)]^{2}\right]} .
\end{aligned}
$$

\section{Acknowledgements}

We thank the Editor and the referee for their comments.

\section{References}

[1] Cvetković, D., Doob, M. and Sachs, H. (1995) Spectra of Graphs—Theory and Application. Barth, Heidelberg.

[2] Gutman, I. (1978) The Energy of a Graph. Ber. Math. Stat. Sekt. Forschungsz. Graz, 103, 1-22.

[3] Gutman, I. (2001) The Energy of a Graph: Old and New Results. In: Betten, A., Kohnert, A., Laue, R. and Wassermann, A., Eds., Algebraic Combinatorics and Applications, Springer-Verlag, Berlin, 196-211. http://dx.doi.org/10.1007/978-3-642-59448-9_13

[4] Gutman, I. (2011) Hyperenergetic and Hypoenergetic Graphs. In: Cvetković, D. and Gutman, I., Eds., Selected Topics on Applications of Graph Spectra, Math. Inst., Belgrade, 113-135.

[5] Gutman, I., Li, X. and Zhang, J. (2009) Graph Energy. In: Dehmer, M. and Emmert-Streib, F., Eds., Analysis of Complex Networks. From Biology to Linguistics, Wiley-VCH, Weinheim, 145-174. http://dx.doi.org/10.1002/9783527627981.ch7

[6] Gutman, I. and Polansky, O.E. (1986) Mathematical Concepts in Organic Chemistry. Springer-Verlag, Berlin. http://dx.doi.org/10.1007/978-3-642-70982-1

[7] Gutman, I. (2005) Topology and Stability of Conjugated Hydrocarbons. The Dependence of Total $\pi$-Electron Energy on Molecular Topology. Journal of the Serbian Chemical Society, 70, 441-456.

[8] Nikiforov, V. (2007) The Energy of Graphs and Matrices. Journal of Mathematical Analysis and Applications, 326, 1472-1475. http://dx.doi.org/10.1016/j.jmaa.2006.03.072

[9] Alwardi, A., Soner, N.D. and Gutman, I. (2011) On the Common-Neighborhood Energy of a Graph. Bulletin T. CXLIII de l'Académie Serbe des Sciences et des Arts 2011 Classe des Sciences Mathématiques et Naturelles Sciences Mathématiques, 143, 49-59.

[10] Koolen, J. and Moulton, V. (2001) Maximal Energy Graphs. Advances in Applied Mathematics, 26, 47-52. http://dx.doi.org/10.1006/aama.2000.0705

[11] Koolen, J.H., Moulton, V. and Gutman, I. (2000) Improving the McClelland Inequality for Total $\pi$-Electron Energy. Chemical Physics Letters, 320, 213-216. http://dx.doi.org/10.1016/S0009-2614(00)00232-3 